Vietnam Journal of Mechanics, VAST, Vol.34, No. 4 (2012), pp. $271-280$

\title{
THE PERFORMANCE IMPROVEMENT OF A LOWCOST INS/GPS INTEGRATION SYSTEM USING THE STREET RETURN ALGORITHM
}

\author{
Nguyen Van Thang1, Pham Manh Thang ${ }^{2}$, Tran Duc Tan² \\ ${ }^{1}$ Broadcasting College 1, Ha Nam, Viet Nam \\ ${ }^{2}$ University of Engineering and Technology, VNU, Hanoi, Vietnam
}

\begin{abstract}
During the last decades, MEMS technology has undergone rapidly development, leading to the successful fabrication of miniaturized mechanical structures integrated with microelectronic components. Accelerometers and gyroscopes are in great demand for specific applications ranging from guidance and stabilization of spacecraft to research on vibrations of Parkinson patient's fingers. The demand of navigation and guidance has been urgent for many years. In fact, INS is used daily in flight dynamics control. Nowadays, with the strong growth of Microelectromechanical system (MEMS) technology, the Inertial Navigation Systems are applied widely. However, there are existing errors in the accelerometer and gyroscope signals that cause unacceptable drifts. Even when the Inertial Navigation System (INS) was supported by the Global Positioning System (GPS), the position error is still large, especially in the case of GPS signal lost. In this paper, we will present a simple algorithm called Street Return Algorithm (SRA) to reduce this kind of error. Experimental result showed that this algorithm could be applied in the real-time operation.
\end{abstract}

Keyword: MicroElectroMechanical Systems (MEMS), Sensor, Inertial Navigation System, Street Return Algorithm (SRA).

\section{INTRODUCTION}

Microelectromechanical systems (MEMS) involve the integration of micro sensors and actuators that sense the environment and react to changes in that environment [1]. They also include the control circuit and the packaging. MEMS may also need micro-power supply and micro signal processing units [2]. MEMS make the system faster, cheaper, more reliable, and capable of integrating more complex functions [3].

In the beginning of the 1990s, MEMS appeared with the development of integrated circuit (IC) fabrication processes. In MEMS, sensors, actuators, and control functions are co-fabricated in silicon. The blooming of MEMS research has been achieved under the strong promotions from both governments and industries. Besides some less integrated MEMS devices such as micro-accelerometers, inkjet printer head, micro-mirrors for projection, etc have been in commercialization; more and more complex MEMS devices have been proposed and applied in such varied fields as microfluidics, aerospace, biomedical, chemical analysis, wireless communications, data storage, display, optics, etc. 
At the end of the 1990s, most of the MEMS transducers were fabricated by bulk micromachining, surface micromachining, and LIthography, GAlvanoforming, moulding (LIGA) processes [4]. Not only silicon but also some more materials have been utilized for MEMS. Furthermore, three-dimensional micro-fabrication processes have been applied due to specific application requirements (e.g., biomedical devices) and higher output power micro-actuators.

Micro-machined inertial sensors that consist of accelerometers and gyroscopes have a significant percentage of silicon based sensors. The accelerometer has got the second largest sales volume after pressure sensor [5]. Accelerometer can be found mainly in automotive industry [6], biomedical application [7], household electronics [8], robotics, vibration analysis, navigation system [9], and so on. Various kinds of accelerometer have increased based on different principles such as capacitive, piezoresistive, piezoelectric, and other sensing ones [10]. The concept of accelerometer is not new but the demand from commerce has motivated continuous researches in this kind of sensor in order to minimize the size and improve its performance.

Let's take a close look at typical application of MEMS-based sensors: navigation and positioning of moving objects. Nowadays, navigation and positioning of the moving objects are important and widely used in civil and military applications [11]. In principle, an Inertial Measurement Unit (IMU) consists of gyroscopes and accelerometers which measure angular velocities and accelerations in three dimensions. Recently, thanks to the development of MEMS technology, the IMUs have become smaller, cheaper and more precise. However, there are still problems with MEMS based on IMUs which are necessary to be solved. The position error of an INS increases rapidly due to the integration of measurement errors in the gyroscopes and accelerometers [12]. To limit these effects on the INS system, a GPS is usually employed simultaneously with an INS. However, the position error of the conventional INS/GPS system in case of GPS outage is still large. Hence, this paper has proposed a new and simple algorithm called Street Return Algorithm (SRA) in order to reduce this error.

\section{MEMS BASED INERTIAL MEASUREMENT UNIT}

An INS consists of angular and linear accelerometers. Angular accelerometers measure how the vehicle is rotating in space and linear accelerometers measure non-gravitational accelerations of the vehicle. Since it can move in three axes, there is a linear accelerometer for each axis. Performing integration on the inertial accelerations using the INS algorithms yields the inertial velocities of the system, and further integration yields the inertial position. With the development of MEMS, those sensors can be produced with low cost, small dimension, low power consumption and fast response allowing continuous update of navigation parameters.

Silicon acceleration sensors generally consist of a proof mass suspended to a reference frame by a spring element. Accelerations cause a displacement of the proof mass, which is proportional to the acceleration. This displacement can be measured in several ways, e.g. capacitive methods measure a change in capacitance between the proof mass and an additional electrode or piezoresistive methods measure resistance change of the 
build-in strain gauges resistors in the spring element [2]. To obtain large sensitivity and low noise, a large proof mass is needed, which suggests the use of bulk micro machined techniques. For less demanding applications surface micro machined devices seem to be more attractive because of the easy integration with electronic circuits and the fact that bulk micromachining requires the use of wafer bonding step [13]. Recently, some designs have been presented which combine bulk and surface micromachining to realize a large proof mass in a single wafer process.

The technology can be classified in a number of ways, such as electromechanical, piezoelectric, piezoresistive, capacitive and electrostatic force balance, resonant accelerometer, etc. Depending on the principles of operations, these accelerometers have their own subclasses. Among them, the capacitive accelerometer is most widely used in micro accelerometer. This paper also focuses on this type of sensors because of its advantages such as high sensitivity, good dc response and noise performance, low drift, low temperature sensitivity, low-power dissipation, and a simple structure.

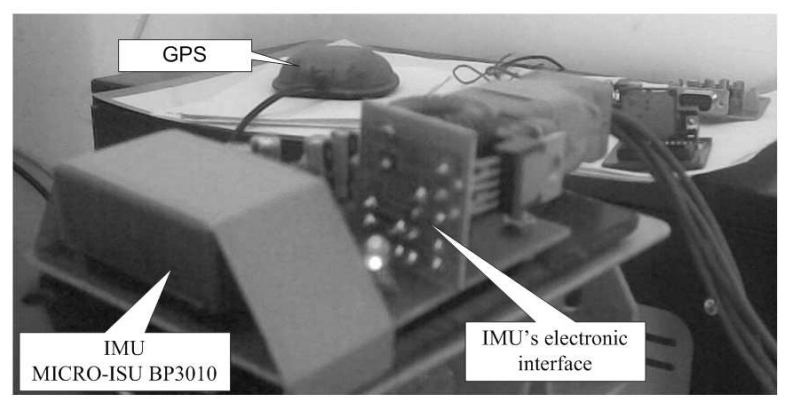

Fig. 1. IMU MICRO-ISU BP3010 and electronic circuits

In this study, we used the MICRO-ISU BP3010 consisting of three ADXRS300 gyros and three heat compensated ADXL210E accelerometers which provide accelerations and angular rates in all 3 vehicle axes [14]. Measuring ranges are $\pm 10 \mathrm{~g}$ and $\pm 300 \% \mathrm{~s}$. The measurements are synthesized by IMU's micro-controllers and transmitted out via RS232 interface. The unit transmits output data as angular and velocity incremental data in serial frames of 16 bytes at one of the user-selectable frequencies of $64 \mathrm{~Hz}, 32 \mathrm{~Hz}, 16 \mathrm{~Hz}$ or $8 \mathrm{~Hz}$. It is a handy little IMU whose dimension is just $35 \times 22 \times 12 \mathrm{~mm}$ (see Fig. 1) and weighing tiny $30 \mathrm{~g}$, with $0.5 \mathrm{~W}$ power consumption.

\section{THE INS/GPS INTEGRATION SYSTEM}

Although INS system is aided by GPS, preliminary valuation worked out that the position error of the INS/GPS system in the case of GPS outage is still large. Therefore, a Filter Kalman (KF) is used for improving this error. The desired parameters are estimated by the Kalman filtering technique applied to the enlarged system. In this integration system, in the input of the Kalman Filter there is the difference between the noisy INS 
output and the noisy GPS output; the output of KF is finally introduced into the unaided INS system. In this structure, INS errors are compensated by a feed forward and/or a feed back loop [15].

Three accelerations $\left(a_{x}, a_{y}\right.$ and $\left.a_{z}\right)$ along three axes of body frame relate to three velocities $(U, V$ and $W)$ in the Earth fixed frame by following equation:

$$
\begin{aligned}
& \dot{U}=a_{x}+V r-W q+g \sin (\theta) \\
& \dot{V}=a_{y}-U r+W p-g \cos (\theta) \sin (\varphi) \\
& \dot{W}=a_{z}-U q+W p-g \cos (\theta) \cos (\varphi)
\end{aligned}
$$

$U, V$ and $W$ are calculated by integrating Eq.1. By using Direct Cosine Matrix $D$, we convert the movement from the Earth fixed frame to the navigation frame:

$$
\left[\begin{array}{c}
\dot{X} \\
\dot{Y} \\
\dot{Z}
\end{array}\right]=\left[\begin{array}{c}
V_{N} \\
V_{E} \\
V_{D}
\end{array}\right]=D^{T}\left[\begin{array}{c}
U \\
V \\
W
\end{array}\right]
$$

We can position the moving object by integrating (2). Then, we obtain the latitude, longitude and height of this object by following equations:

$$
\dot{\lambda}=\frac{V_{N}}{R_{\text {earth }}}, \quad \dot{\mu}=\frac{V_{E}}{R_{\text {earth }} \cos (\lambda)}, \quad \dot{H}=-V_{D}
$$

The GPS consists of 24 satellites which fly above the surface of the Earth at the height of $19,200 \mathrm{~km}$ in order to acquire the position of the moving objects (latitude, longitude and height). Radio signals hardly get through solid buildings, tunnels, etc. To get the correct position of the moving object it requires at least four satellites available.

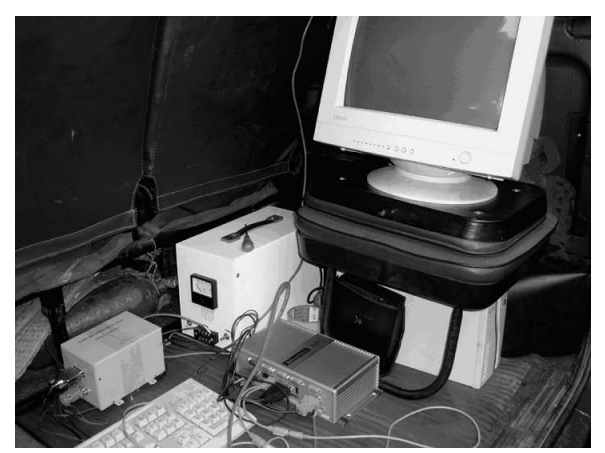

Fig. 2. The INS/GPS system in outdoor experimentation 
The Kalman filter is a digital filter that can optimally estimate in real time the states of the system based on its noisy outputs. These states are position errors and velocity errors of the INS. The GPS output is used as a tool to estimate the error in the INS and to correct the error as much as possible. It is called the GPS-aided INS system configuration [15]. An INS/GPS integrated system was developed successfully in our lab (see Fig. 2). However, in the case of GPS outage, the integrated system still makes position errors of tens of meters in a short time (approx. $70 \mathrm{~s}$ ) [16]. It will be illogical when the vehicle is running on the roads but the result shows that it is running on the street side. Thus, in this paper, a street return algorithm (SRA) has been developed in order to minimize the position error when GPS outage.

\section{THE PROPOSED INS/GPS/SRA INTEGRATED SYSTEM}

The previous section presented about INS/GPS system configuration and preliminary analysis about the advantage and disadvantage of this configuration. However, we need to impress that the role of GPS in this system is very important. Therefore, when GPS outage, this system will work ineffectively. In order to overcome the disadvantage, we proposed the street return algorithm (SRA) and embedded in an INS/GPS integrated system. In this study, we assumed that the vehicle only runs on certain streets whose location information is stored in the database.

This proposed system is shown in Fig. 3.

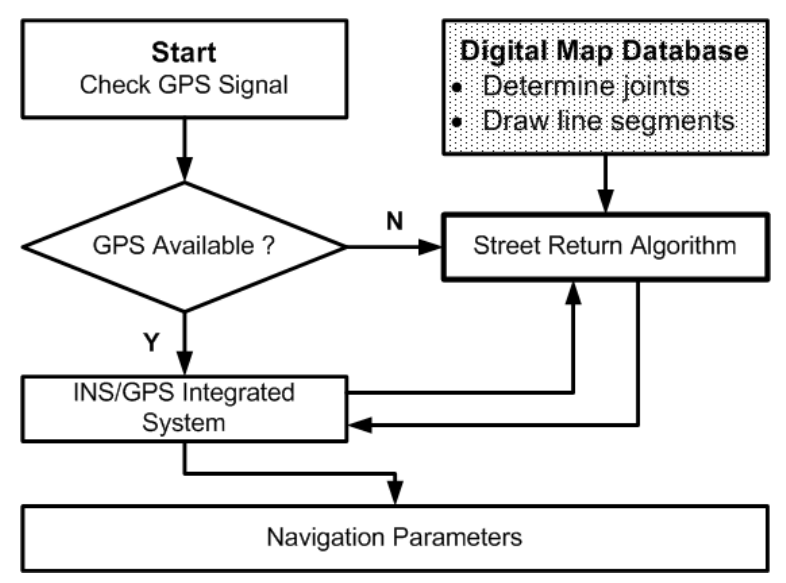

Fig. 3. Diagram of the proposed system

The implementation of system can be divided into three steps:

Step 1: From the trajectory of proposed roads, we use digital map database to select some joints (with their latitude and longitude coordinates) on those roads. After that, we can determine lines which run through these joints.

In order to determine a line past two joints $\mathrm{A}\left(x_{A}, y_{A}\right)$ and $\mathrm{B}\left(x_{B}, y_{B}\right)$, we use the following formula: 


$$
y=\frac{y_{B}-y_{A}}{x_{B}-x_{A}} x+y_{A}-\frac{y_{B}-y_{A}}{x_{B}-x_{A}} x_{A}
$$

After being created, the trajectory of the proposed roads becomes the trajectory of line segments (as shown in Fig. 4). This step is implemented in the database block.

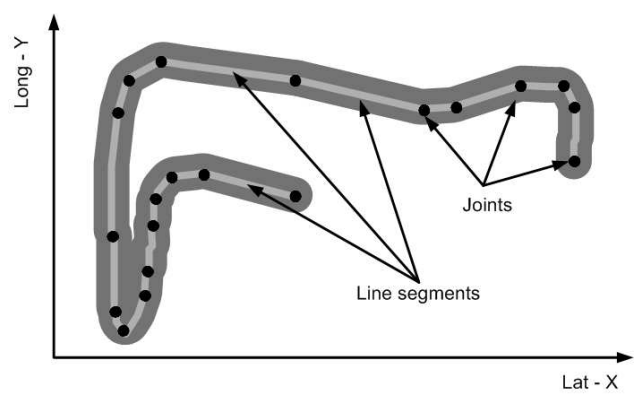

Fig. 4. Determination of joints and linear segments road

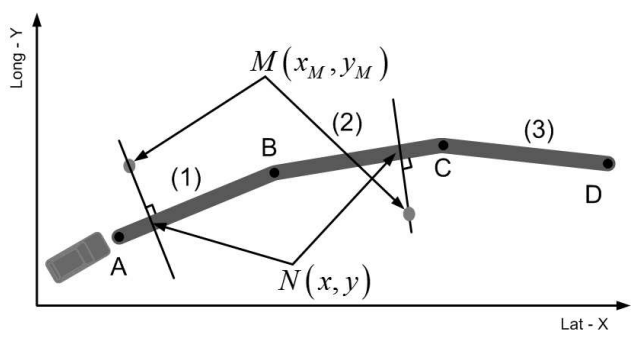

Fig. 5. Determination the nearest coordinate to replace the inaccurate one

Step 2: If GPS signal is available, location information of GPS is directly taken to the INS/GPS integrated system. In case that the GPS signal is not available, location information computed by INS in INS/GPS integrated system is brought to SRA block. The main task of this block is to estimate the deviation between the real coordinate and the coordinate provided by INS, and find out the most suitable location of the vehicle. When the land vehicle moves on a specific road segment, the most suitable location of the vehicle is the nearest location on the road segment (see Fig. 5). In this figure, $\left(x_{M}, y_{M}\right)$ are latitude and longitude coordinates calculated by INS when there is no GPS signal, and $(x, y)$ are coordinates found out in order to replace $\left(x_{M}, y_{M}\right)$.

To determine $(x, y)$, we suppose that the land vehicle is moving on the first segment AB. When GPS signal is not available, if INS indicates that location of the land vehicle at a specific time is point $M$ (blue point), we can calculate the distance between two points $\mathrm{M}$ and $\mathrm{N}$. In addition, $(x, y)$ coordinates will be found out in order to replace $\left(x_{M}, y_{M}\right)$ coordinates.

From point M, we create a perpendicular to segment AB. This perpendicular line has the equation:

$$
\left(x-x_{M}\right)+\frac{y_{B}-y_{A}}{x_{B}-x_{A}}\left(y-y_{M}\right)=0
$$

Therefore, $(x, y)$ can be calculated by:

$$
\begin{aligned}
& \left(x-x_{M}\right)+\frac{y_{B}-y_{A}}{x_{B}-x_{A}}\left(y-y_{M}\right)=0 \\
& \frac{y_{B}-y_{A}}{x_{B}-x_{A}} x+y_{A}-\frac{y_{B}-y_{A}}{x_{B}-x_{A}} x_{A}-y=0
\end{aligned}
$$


Then obtain:

$$
\begin{aligned}
& x=-\frac{y_{B}-y_{A}}{x_{B}-x_{A}}\left(y-y_{M}\right)+x_{M} \\
& y=\frac{\left(y_{B}-y_{A}\right)\left(x_{B}-x_{A}\right)\left(x_{M}-x_{A}\right)+\left(y_{B}-y_{A}\right)^{2} y_{M}+\left(x_{B}-x_{A}\right)^{2} y_{A}}{\left(y_{B}-y_{A}\right)^{2}+\left(x_{B}-x_{A}\right)^{2}}
\end{aligned}
$$

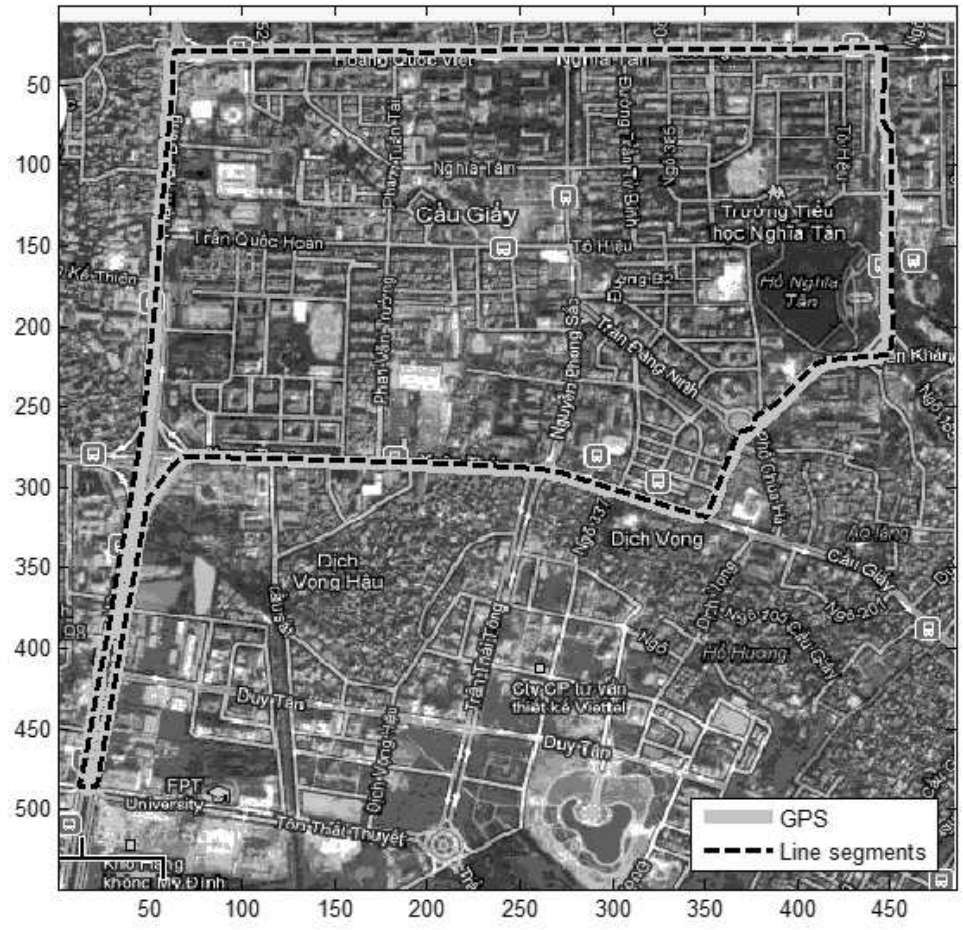

Fig. 6. Navigation map, line segments based trajectory, and GPS based trajectory

We represent the distance from $\mathrm{M}$ to $\mathrm{N}$ as $d$, then $d$ is the deviation between real coordinates and incorrect coordinates. Using Haversine formula [17], we applied the following program to calculate the distances between two points from their longitudes and latitudes:

When the land vehicle moves past the $B$ point, the determination $(x, y)$ and $\mathrm{d}$ is the same on the $A B$ line segment.

Step 3: Display the navigation parameters: positions $(x, y, z)$, velocities $(U, V, W)$ and attitudes $(\phi, \theta, \varphi)$. This step is implemented in INS/GPS integrated system block.

\section{RESULTS AND DISCUSSIONS}

This proposed system and algorithm is characterized for vehicle navigation in Hanoi, Vietnam. A closed trajectory of Nguyen Van Huyen - Hoang Quoc Viet - Pham Van 
Program 1. Haversine for formula

$\mathrm{R}=$ earth's radius (mean radius $=6.371 \mathrm{~km}$ )

$\Delta_{l a t}=\left(x_{M}-x\right) * p i / 180$

$\Delta_{\text {long }}=\left(y_{M}-y\right) * p i / 180$

$a=\sin ^{2}\left(\Delta_{\text {lat }} / 2\right)+\cos (x) * \cos \left(x_{M}\right) * \sin ^{2}\left(\Delta_{\text {long }} / 2\right)$

$c=2 * \operatorname{atan} 2(\operatorname{sqrt}(a), \operatorname{sqrt}(1-a))$

$d=R * c$

Dong - Pham Hung - Xuan Thuy - Tran Dang Ninh - Nguyen Khanh Toan is used for an initial evaluated process. Fig. 6 shows the navigation map, line segments based trajectory and GPS based trajectory. Normally, general road, especially interstate road and highway is compounded of several line segments. In stead of developed road data base, this work proposed a novel method to build a trajectory based on connection of the line segments. Therefore, the used closed trajectory is divided by 57 joints.

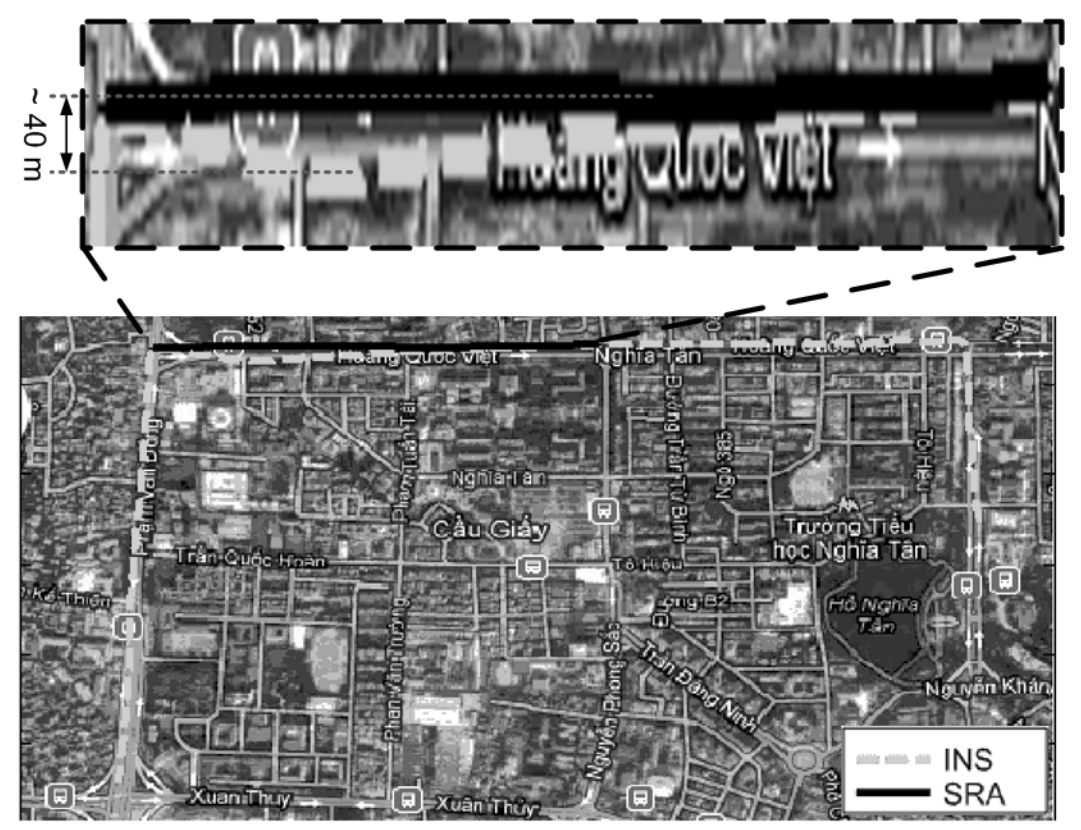

Fig. 7. Output positions of the INS system and the SRA integrated system

In this experimental work, GPS signal is assumed lost within 100 seconds while land vehicle crosses the Hoang Quoc Viet Street. The maximum deviation that the INS/GPS system calculated is about 40 meters without SRA (see Fig. 7). The trajectory of line segments and results of the proposed INS/GPS/SRA integration system are also shown in Fig. 8. The proposed SRA results are entirely met the line segments based trajectory which is built by simply linear connecting 57 joints in Fig. 6 . In this initial characterizing, 


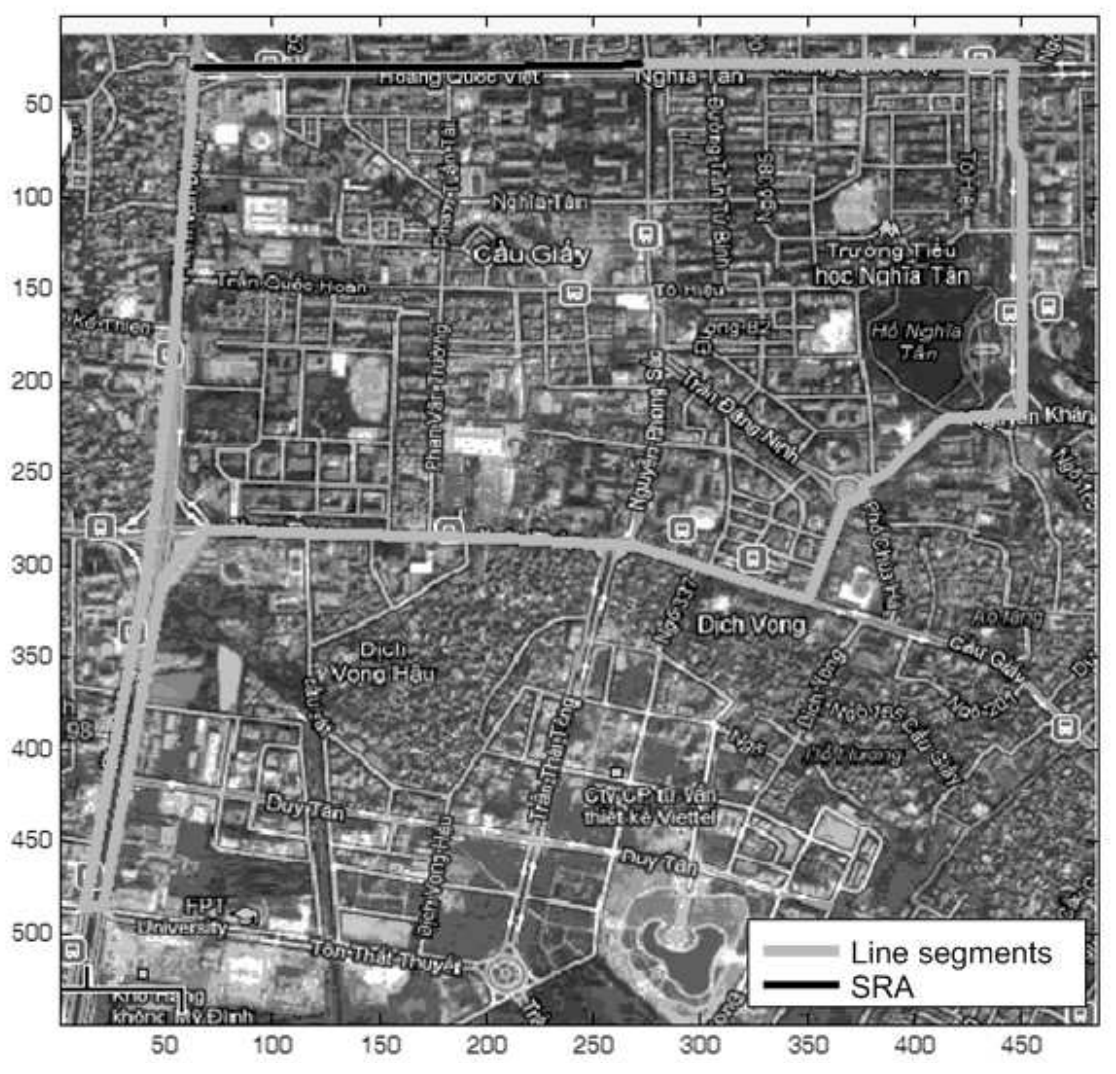

Fig. 8. Land vehicle's trajectory

the joints are simple selected at the center of the second lane. The calculated results shows that the output deviation of this SRA system is about \pm 1 meter in the transverse direction while the best GPS error of about \pm 5 meter [18].

\section{CONCLUSION}

GPS outage is a serious problem for any INS/GPS integration system. This paper has succeeded in embedding the street return algorithm into a conventional low-cost INS/GPS integration system [16] in order to reduce the vehicle's drift when it falls off the street due to GPS outage. The simulated results show that the proposed system offers a deviation within one meter compared to true transverse locations. Moreover, this is a simple algorithm, so, it can be easily implemented to the conventional real time system.

\section{ACKNOWLEDGMENT}

This work is partly supported by the project QG-11.31. 


\section{REFERENCES}

[1] Middelhoek S., Noorlag D. J. W., (1992), Signal conversion in solid-state transducers, Sensors and Actuators, Vol. 2, pp. 211-228.

[2] Danny Banks, Microengineering, MEMS, and Interfacing: A Practical Guide, CRC Press, (2006).

[3] Cimoo Song, Commercial vision of silicon based inertial sensors, TRANSDUCERS'97, Vol. 2, Chicago, (1997), pp.839-842.

[4] Cornelius T. Leondes el at, MEMS/NEMS Handbook Techniques and Applications, Springer Press, (2006).

[5] Roylance L.M., Angell J.B, A batch-fabricated silicon accelerometer, IEEE Transactions on Electron Devices Vol. 26(12), (1979), pp.1911-917.

[6] Spangler C., Kemp C.J., Integrated silicon automotive accelerometer, Sens. Actuators A54, (1996), pp. 523-529.

[7] Hoff L., Elle O.J., Grimnes M., Halvorsen S., Alker H.J., Fosse E., (2004), Measurements of heart motion using accelerometers, Proceedings of IEEE Sensors Vol. 3, pp. 1353-1354.

[8] T.Velten, P. Krause, E. Obermeier, (1996), Two-axis Micromachined Accelerometer for Gesture Recognition, $M M E^{\prime} 96$, pp. 247-250.

[9] Santiram Kal, Design and Fabrication of MEMS Accelerometer for Avionics Applications, Proceedings of International Conference on Smart materials Structures and Systems, Bangalore, India, (2005), pp. 140-146.

[10] Garcia Valenzuela and M. Tabib-Azar, Comparative study of piezoelectric, piezoresistive, electrostatic, magnetic, and optical sensors, Proc. SPIE, USA, (1994), pp. 125-142.

[11] Santiram Kal, Design and Fabrication of MEMS Accelerometer for Avionics Applications, Proceedings of International Conference on Smart materials Structures and Systems, Bangalore, India, (2005), pp. 140-146.

[12] T. D. Tan, L. M. Ha, N. T. Long, N. D. Duc, N. P. Thuy, Integration of Inertial Navigation System and Global Positioning System: Performance analysis and measurements, International Conference on Intelligence and Advance Systems 25th - 28th November 2007 KL Convention Center, Kuala Lumpur, Malaysia, pp.1047-1050.

[13] Bernhard E. Boser, Roger T. Howe, Surface Micromachined Accelerometer, IEEE Journal of solid - state circuits, Vol. 31(3), (1996), pp. 179-191.

[14] Georey J.Bulmer, In MICRO-ISU BP3010 An OEM Miniature Hybrid 6 Degrees-Of-Freedom Inertial Sensor Unit. Gyro Symposium, Stuttgart 16th-17th September, (2003).

[15] Tran Duc Tan, Luu Manh Ha, N. T. Long, H. H. Tue, N. P. Thuy, Novel MEMS INS/GPS Integration Scheme Using Parallel Kalman Filters, Proceedings of the 2008 IEEE International Symposium on System Integration, Japan, December (2008), pp. 72-76.

[16] Tran Duc Tan, Luu Manh Ha, Nguyen Thang Long, Nguyen Dinh Duc, Nguyen Phu Thuy, Land-Vehicle MEMS INS/GPS Positioning During GPS Signal Blockage Periods, Journal of Science VNUH, Vol. 23, No. 4, (2007), pp. 243-251.

[17] R. W. Sinnott, Virtues of the Haversine, Sky and Telescope, 68 (2), (1984), p. 158.

[18] HICOM USB GPS data sheet (http://www.haicom.com.tw/HI-204III-USB)

Received July 31, 2012 